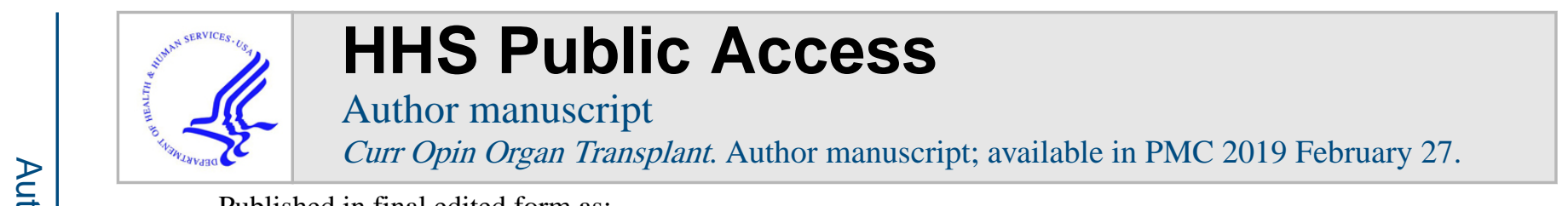

Published in final edited form as:

Curr Opin Organ Transplant. 2017 December ; 22(6): 541-548. doi:10.1097/MOT.0000000000000465.

\title{
Lung xenotransplantation
}

\author{
Hisashi Saharaa ${ }^{a, b}$, Hironosuke Watanabe ${ }^{a}$, Thomas Pomposellia , and Kazuhiko Yamada ${ }^{a}$ \\ ${ }^{a}$ Columbia Center for Translational Immunology, Columbia University Medical Center, New York, \\ New York, USA \\ bDivision of Organ Replacement and Xenotransplantation Surgery, Center for Advanced \\ Biomedical Science and Swine Research, Kagoshima University, Kagoshima, Japan
}

\begin{abstract}
Purpose of review-This review describes the most recent progress in xeno lung transplantation (XLTx) to date. It describes the potential mechanisms of early xeno lung graft loss, as well as the latest therapeutic strategies to overcome them.
\end{abstract}

Recent findings-Using ex-vivo perfusion models of porcine lungs with human blood, the use of genetically modified pig lungs along with novel pharmaceutical approaches has recently been studied. Strategies that have demonstrated improved lung survival include the knockout of known xenoantigens (GalTKO and N-glycolylneuraminic acid-KO), genes that regulate complement activation (hCD46 and hCD55), as well as the inflammation/coagulation cascade (human leukocyte antigen-E, human thrombomodulin, human endothelial protein $\mathrm{C}$ receptor, hCD47, hCD39, hCD73 and heme oxygenase-1). Furthermore, pharmacologic interventions including the depletion of pulmonary intravascular macrophages or von Willebrand factor, inhibition of thromboxane synthase and blockade of histamine receptors have also demonstrated protective effects on xeno lung grafts. Using in-vivo pig to nonhuman primate lung transplant models, these approaches have been shown to extend pulmonary xenograft survival to 5 days.

Summary-The development of new multitransgenic GalTKO pigs has demonstrated prolongation of porcine xenograft survival; however, advancement in XLTx has remained frustratingly limited. Further intensive and innovative strategies including genetic manipulation of donors, as well as inflammation/coagulation dysregulation, are required to make XLTx a clinical possibility.

\section{Keywords}

endothelial injury; genetical modification; inflammation/coagulation dysregulation; lung transplantation; xenotransplantation

Correspondence to Kazuhiko Yamada, MD, PhD, Columbia Center for Translational Immunology, Columbia University Medical Center, 630 W 168th St, BB1705, New York, NY 10032, USA. Tel: +1 212304 5695; ky2323@ cumc.columbia.edu.

Conflicts of interest

There are no conflicts of interest. 


\section{INTRODUCTION}

Despite decades of research, lung transplantation (LTx) continues to be the only definitive treatment for many chronic respiratory illnesses. The major obstacle that LTx faces, however, is the paucity of viable organs available for transplant. As of 2012, donor lung utilization rates hovered at only $37 \%$ [1], indicating a major clinical disparity between the number of patients awaiting LTx and available donor lungs. This is due largely in part to preexisting lung disease in deceased donors, which include pneumonia, acute lung injury and chest trauma [2]. Because of this, there is an ever-increasing need for alternative sources of organs for patients awaiting lung transplant. Xenotransplantation, or inter-species transplantation, is a potential solution, as it would offer the benefit of a potentially limitless supply of organs.

Recently, several groups have demonstrated promising results with marked survival of genetically engineered porcine organs in nonhuman primates (NHPs) [3 $\left[\mathbf{m}_{, 4,5,6}, 7 \mathbf{m}^{\mathbf{m}}\right.$ ]. However, progress in the field of xeno lung transplantation (XLTx) has been frustratingly limited, with only moderate clinical successes and survival rates measured in days [ ${ }^{\mathbf{m}}$ ]. The reasons for this are most certainly multi-factorial, and are likely because of multiple immunologic and nonimmunologic barriers unique to the lung. This review describes the recent progress in XLTx to date, describes some of the aforementioned barriers, as well as our strategies to overcome them.

\section{TEXT OF REVIEW}

\section{Xeno lung transplantation in small animal models: greater than 3 months survival of orthotopic lung transplantation in hamster to rat models}

One of the earliest demonstrations of XLTx was in 1998, in which Miyata et al. [9] demonstrated inter-species graft survival of over 3 months in a hamster to rat model [10,11]. Miyata et al. [9] performed XLTx using Golden Syrian hamsters as donors and Lewis rats as recipients, followed by a short course of tacrolimus and cyclophosphamide in their experimental groups. They reported that after left LTx was performed, the rats that were treated with tacrolimus and cyclophosphamide had a median graft survival time (MST) of 96.5 days, whereas MST of control animals without treatment was 3 days.

\section{Xeno lung transplantation in pig to nonhuman primates}

Even though Miyata's et al. [9] results were promising, the outcomes of larger animal models pertaining to XLTx have remained limited. Although there has been marked improvement in the survival of various solid organ xenografts in pig-to-NHP models [3 $3^{\mathbf{m}}$, $4,5,6 \mathbf{\square}, 7$ ], the longevity of xeno lung grafts in these large animal models is brief with only sporadic reports of survival beyond 3 days [12]. Burdorf et al. [8] suggested that slightly better outcomes may be possible by utilizing porcine lungs from multitransgenic ( $\mathrm{Tg}$ ) donors. In this study, even though the recipient animals survived until postoperative day (POD) 8, no histologic evidence was presented to show the health of the graft beyond POD 3. At time of necropsy, the authors found completely rejected porcine lungs. The longest survival with a histologically proven lung graft at POD 5 in a baboon, however, was reported 
by Cantu et al. [13] in 2007 in which the authors presented evidence of a viable lung graft on POD 5. The authors had previously demonstrated the key role that swine pulmonary intravascular macrophages (PIMs) play in the hyper-acute dysfunction and consumptive coagulopathy that is characteristic of lung xenotransplant. In addition to their scavenging function, PIMs produce a number of proinflammatory cytokines and procoagulant factors. Cantu et al., using an in-vivo pig to primate orthotopic LTx model after PIM depletion, were able to abrogate the consumptive coagulopathy seen in controls and extend graft function to $24 \mathrm{~h}$. The authors sought to evaluate whether the depletion of von Willebrand factor (vWF) along with the absence of PIMs would have an additive effect on pulmonary xenograft survival. Utilizing a left single-LTx model, baboons depleted of antiaGal antibodies received lungs from either $v W F-d e f i c i e n t(n=2)$, human membrane cofactor protein (hMCP)-expressing ( $n=5)$, hMCP PIM-depleted $(n=5)$ or vWF-deficient PIM-depleted swine $(n=3)$. Two out of three of the PIM-depleted, vWF deficient grafts survived up to 5 days. Depletion of PIMs from vWF-deficient lungs, like depletion of PIMs from hMCP lungs, resulted in abrogation of the coagulopathy associated with pulmonary xenotransplantation. This is most likely because of the interplay between the inflammatory response and the coagulation cascade witnessed with acute lung rejection. Their experiment supported the ex-vivo perfusion data [14] that vWF plays a role in the 'delayed' (24 h) dysfunction observed in pulmonary xenotransplantation using PIM-depleted hMCP swine lung.

Figure 1 illustrates a potential cascade of the early xeno lung graft loss associated with endothelial injury (Fig. 1). Recent studies have shown that the vasculature of the lung xenografts releases larger quantities of vWF compared to cardiac or renal xenografts [15], porcine vWF binds to human or NHP Glycoprotein Ib (GPIb) on platelets even in the absence of shear stress, resulting in abnormal platelet activation and aggregation following XLTx[16]; GPIb blockade significantly reduced platelet activation and delayed platelet sequestration. This effect was amplified by pretransplant depletion of vWF from pig lung using 1-deamino-8-d-arginine vasopressin (DDAVP) [17].

\section{Ex-vivo lung xeno-perfusion models of porcine lungs to determine possible mechanisms of the early loss of xenolung grafts}

In order to determine the mechanisms of early graft loss of porcine lungs in NHP, the Maryland group and others have performed studies using models consisting of porcine exvivo lungs perfused with human blood. The aim is to evaluate the effects of genetically modified pig lungs, as well as pharmaceutical approaches on preventing rapid xeno lung rejection [18].

\section{GalTKO or hCD46/GalTKO lungs}

When wild-type swine lungs are perfused with human blood, the grafts demonstrate rapid respiratory failure within 10-20 min. In contrast, GalTKO lungs are noted to survive for over 120 min when perfused with human blood [19-21]. Platelet sequestration, a known sign of acute rejection, was also diminished in GalTKO lungs relative to wild-type lungs. In addition to GalTKO, the role of human complement regulatory protein hCD46 was also investigated. In hCD46/GalTKO lungs, longer MST was observed compared to GalTKO 
lungs (171 min for hCD46/GaTKO vs. $120 \mathrm{~min}$ for GalTKO) [22]. There were also diminished platelet and coagulation cascade activation, neutrophil sequestration and histamine release within the first 120 min of perfusion.

\section{Human thrombomodulin/hCD46/GalTKO or human endothelial protein C receptor/hCD46/ GalTKO lungs}

As eluded to previously, incompatibility between pig and human proteins related to the interplay between inflammation and the coagulation cascade is one of the key factors of early lung xenograft failure. Human thrombomodulin (hTBM) is expressed on vascular endothelium and binds to human thrombin to act as an anticoagulant. Thereafter, this hTBM/ thrombin complex activates protein $\mathrm{C}$ to produce activated protein $\mathrm{C}$, which possesses antiinflammatory and cytoprotective activities via human endothelial protein $\mathrm{C}$ receptor (hEPCR) [23]. However, pig TBM is a poor regulator of human thrombin, leading to the development of microvascular thrombosis as well as severe inflammatory responses in pigto-primate xenografts [24]. Harris et al. [25 ${ }^{\mathbf{D}}$ ] hypothesized that by adding hTBM or hEPCR to the genetic background of transgenic hCD46/GalTKO swine, this would have a protective effect against the complement-driven rejection of lung xenografts. Unfortunately, hTBM/ hCD46/GalTKO lungs did not show significant clinical effect when used in an ex-vivo lung xeno-perfusion model, although the additionally expressed hEPCR on hCD46/GalTKO did slightly prolong the lung xenograft MST up to $240 \mathrm{~min}$ from $171 \mathrm{~min}$ [26]. The authors suggested that to optimize the activation of human protein C, coexpression of hTBM and hEPCR may be required [25 ${ }^{\boldsymbol{m}}$.

\section{hCD39/GalTKO lungs}

Inflammatory mediator generation is an additional key therapeutic target in XLTx. hCD39 is a cell surface molecule that converts adenosine triphosphate (ATP) and adenosine diphosphate (ADP) to adenosine monophosphate (AMP), which is then converted to adenosine by hCD73 [27]. The net effect is removal of the proinflammatory signal ATP and the platelet agonist ADP, and generation of adenosine, which possesses anti-inflammatory effects [27]. It has been hypothesized that the expression of hCD39 and/or hCD73 in donor pigs would provide both thromboregulatory and anti-inflammatory effects [28]. Unfortunately, the effects of hCD39 expression on pulmonary xenograft survival have been inconclusive to date $\left[20,25^{-}\right.$], and further studies are required to elucidate the exact role that expression of CD39 on pulmonary xeno-grafts would play in extending their survival. This would involve additional studies of coexpression of hCD73 to enhance the hydrolysis of extracellular AMP to adenosine [29].

\section{Heme oxygenase-1/hCD46/GalTKO lungs}

Heme oxygenase-1 (HO-1) is an inducible enzyme and a fundamental sensor of cellular stress and directly contributes toward limiting or preventing tissue damage [30]. Expression of hHO- 1 on hCD46/GalTKO has been shown to reduce inflammation and improve pulmonary xenograft survival in ex-vivo perfusion models [25 ${ }^{\mathbf{m}}$ ]. 


\section{hHLA-E/hCD46/GalTKO lungs}

Natural killer (NK) cell activation is negatively regulated by binding to human leukocyte antigen-E (HLAE) [31], and Laird et al. [32] had hypothesized that hCD46/GalTKO pig lungs genetically modified to express HLA-E would be protected against NK cell-mediated injury and display prolonged lung function. In fact, they were able to show that relative to hCD46/GalTKO pig lungs perfused with human blood on an ex-vivo platform, additional expression of HLA-E increased MST from 162 min to more than $240 \mathrm{~min}$.

\section{$\mathrm{N}$-glycolylneuraminic acid-KO lungs}

During ex-vivo perfusion with human blood, GalTKO swine lungs survive approximately 2 $\mathrm{h}$ relative to wild-type ( $<15 \mathrm{~min}$ ) [19], whereas in-vivo life-supporting lung transplants in baboons have been shown to survive on average $215 \mathrm{~min}$ (described below in-vivo section) [33]. N-glycolylneuraminic acid (NeuGc) is one of the most significant xenoantigens in GalTKO organs. Recent studies have examined the role that preformed human antibodies directed at Neu5Gc play on xeno lung graft failure. These studies have demonstrated that human serum anti-NeuGc IgM titers decreased approximately $40 \%$ during the ex-vivo perfusion of hCD46/GalTKO lungs, whereas anti-NeuGc IgM titers did not fall during the perfusion of NeuGcKO lungs [34].

\section{Other pharmacologic interventions}

Recent studies have demonstrated that thromboxane synthase inhibition along with histamine receptor blockade improves ex-vivo hCD46/hCD55/GalTKO pig lung function. In contrast, control lungs have shown a pulmonary vein resistance (PVR) rise within the first hour of perfusion (mean PVR at $30 \mathrm{~min}: 286 \pm 83 \mathrm{mmHg} / \mathrm{min} / \mathrm{l}$ ), whereas treated lungs exhibited no rise in PVR throughout the experiment (33 \pm 3 at $30 \mathrm{~min}$ ) [35].

\section{In-vivo pig-to-nonhuman primate lung transplant models: overcome the limitation of ex- vivo lung xeno-perfusion models}

Although ex-vivo lung xeno-perfusion models have the advantages of allowing direct study of human antipig responses both mechanically and physiologically [18], this model is limited to only short-term effects (usually within $4 \mathrm{~h}$ ). Another short coming of this study design is that exposure of blood to the artificial surfaces of the ex-vivo perfusion circuit along with the concomitant intensive anticoagulation response could introduce potentially confounding variables [33]. Because of this, ex-vivo perfusion models may be significantly flawed, and in order to establish the long-term evaluation of lung injury and performance studies utilizing porcine orthotopic, XLTx in NHP is critical.

\section{GalTKO lung in baboons}

In 2007, Nguyen et al. were [33] the first to demonstrate the in-vivo protective effects of the GalTKO transgene on lung xenografts. In their study, three GalTKO left swine lungs were compared with two swine lung xenografts transgenic for hCD46 in baboons in a lifesupporting model. Two of three GalTKO lungs supported life for 90 and $215 \mathrm{~min}$, and displayed low PVR (48 $\pm 12 \mathrm{mmHg} \times \mathrm{min} / \mathrm{l}$ at $60 \mathrm{~min})$, whereas hCD46 Tg lungs exhibited high PVR (> $500 \mathrm{mmHg} \times \mathrm{min} / \mathrm{l})$ and failed to support life by the $21 \mathrm{st}$ min of the study. 
GalTKO swine lungs seem to have some protective effect in vivo from the physiologic consequences associated with hyperacute lung rejection. However, it is apparent that GalTKO itself is not sufficient to protect grafts from early rejection characterized by increased pulmonary vascular resistance, capillary leak and endothelial injury.

\section{hCD46/GalTKO lungs in baboons}

In accordance with the progress of the latest gene-editing technology, an increasing number of genetically engineered pigs are becoming available [36-38]. Burdorf and Pierson [39] have performed life-supporting lung transplants in baboons using hCD46/GalTKO porcine lung grafts. The addition of hCD46 had almost no effect on prolonging graft survival times, with the average time of viability having a median of $240 \mathrm{~min}$, range 0-240 min.

\section{hCD55/GalTKO lungs in baboons}

The rapid development of disseminated intravascular coagulation (DIC) is a well-known hindrance to the success of pig to baboon XLTx. Bush et al. [40] sought to show that porcine GalTKO lungs that also expressed the complement regulatory protein hCD55 would prevent this consumptive coagulopathy. For their study, the group utilized two baboons that were depleted of macrophages in a nonlife-supporting model. Orthotopic transplant of the left lung was then carried out using hCD55/GalTKO swine lungs. Both cases developed DIC and lost grafts within $48 \mathrm{~h}$ of reperfusion ( 3.5 and $48 \mathrm{~h}$ ) with associated thrombocytopenia.

\section{hCD55/human endothelial protein C receptor/hCD47/hTFP/hCD46/GalTKO lungs in baboons}

XLTx was performed using swine lungs with six genetic modifications (hCD55/hEPCR/ hCD47/hTFP/hCD46/GalTKO). The grafts were then treated with DDAVP, followed by the treatment with an extensive pharmacologic and immunologic regimen. This included pretreatment with steroids, $\mathrm{C} 1$ esterase inhibitor, heparin, thromboxane synthase inhibitor, histamine receptor blockers, anti-GPIb Fab and immunosuppression including antithymocyte globulin, Mycophenolate mofetil and tacrolimus or anti-CD40 antibody. The authors reported that extubation was successfully performed in 54\% (seven of 13) of the cases, and within these seven cases the posttransplant survival of the recipients ranged from 1 to 8 days, although no histo-logical data were presented to demonstrate the survival of the grafts beyond POD $3\left[8^{\mathbf{m}}\right]$.

\section{Summary of published data on in-vivo pig-tononhuman primate xeno lung transplantation}

To date, the most commonly used in-vivo XLTx model involves a left single lung xenograft that is orthotopically transplanted into an NHP. Thus far, the longest survival with histologically proven viable lung grafts in baboons has been reported by Cantu et al. [13] in 2007 in which they used membrane cofactor protein Tg pigs as donors (Table 1) [8 , $13,33,39,40]$. Since then, multiple Tg to GalT-KO studies have been conducted; however, none of them to date has been able to avoid the very early acute rejection response. Pig xeno lungs have proven to be highly susceptible to acute humoral rejection in which the fragile alveolar capillary endothelium is able to be damaged by preformed natural antibodies (nAb) even at low levels. Because of the robust innate and adaptive immunologic response, as well 
as pig xeno lung's susceptibility to preformed antipig antibodies, innovative strategies must be developed to overcome the many barriers to making pig lungs a viable option for xenotransplantation.

\section{Our recent data and strategies to overcome the early loss of xeno lung grafts}

Despite the use of multi-Tg pigs as donors, results of XLTx remain limited (Table 1). Therefore, it is clear that further strategies, besides muti-Tg donors, are required to prolong the paltry lung graft survival from days to months. Among the strategies we are currently exploring for this purpose include a novel means of minimizing the inflammatory response caused by preformed $\mathrm{nAb}$, macrophages, $\mathrm{NK}$ cells, $\mathrm{T}$ cells and $\mathrm{B}$ cells before transplantation. An operative strategy that we are developing involves cotransplanting a vascularized thymic graft along with the lung from the same swine donor. This is a strategy we have recently utilized in a baboon recipient of a pig kidney that has achieved greater than 6-month survival, along with evidence of recipient donor-specific unresponsiveness at both T-cell and B-cell levels $\left[6^{\mathbf{m}}\right.$ ]. Another technique involves the use of intrabone bone marrow transplantation. Our prior work with this technique has allowed pig bone marrow cells to engraft for over 28 days in a baboon, which resulted in the induction of donor-specific unresponsiveness in vitro and prolonged survival of a xenokidney [41]. Finally, we are using a technique that has markedly improved ischemia-reperfusion injury of swine lungs, as well as improved their survival across allogeneic barriers that involves using a lung-specific carbon monoxide inhalation strategy $[42,43]$. We believe that the application of these strategies are the crucial next steps in progressing the field of XLTX, and they will result in overcoming the unique immunologic barriers that XLTx presents.

\section{CONCLUSION}

According to the Registry of the The International Society for Heart \& Lung Transplantation, the 5-year patient survival for allogeneic lung transplant recipients is only $54 \%$, with as many as $21 \%$ of these patients dying from acute rejection or graft failure. In addition, 29\% of surviving lung transplant recipients require treatment for acute allograft rejection within a year of their transplant. [44]. With such poor success rates with allogeneic lung transplants, it comes as little surprise that successful lung transplants from differing species would present an even greater level of difficulty. It has been shown that the unique structure of swine alveolar capillary endothelium is particularly susceptible to preformed antipig antibodies that are generated across species barriers. The immunoresponses that are associated with species incompatibility serve to severely damage the endothelium of capillary loops of pig lungs, resulting in the accelerated acute xenograft humoral rejection seen in primates after transplant.

The development of new multitransgenic GalTKO pigs has demonstrated marked prolongation of porcine xenograft survival after orthotopic transplant into NHP. However, advancement in XLTx has remained frustratingly limited. As reviewed in this article, the lung possesses several unique immunologic and nonimmunologic barriers that continue to stifle progress in the field of XLTx. Intensive and innovative strategies such as genetic 
manipulation of donors, coagulation dysregulation and the development of novel therapies to induce tolerance must be developed to make XLTx a clinical possibility.

\section{Acknowledgements}

The authors would like to thank Dr David H. Sachs for his helpful advice and review of this article.

Financial support and sponsorship

This research was supported by a Grant-in-Aid for Scientific Research (B) (H.S.; Grant Number 16H05432).

\section{REFERENCES AND RECOMMENDED READING}

Papers of particular interest, published within the annual period of review, have been highlighted as:

of special interest

m of outstanding interest

1. Valapour M, Skeans MA, Heubner BM, et al. OPTN/SRTR 2012 Annual data report: lung. Am J Transplant 2014; 14(Suppl 1):139-165. [PubMed: 24373171]

2. Laubach VE, Sharma AK. Mechanisms of lung ischemia-reperfusion injury. Curr Opin Organ Transpl 2016; 21:246-252.

3. Higginbotham L, Mathews D, Breeden CA, et al. Pretransplant antibody screening and anti-CD154 costimulation blockade promote long-term xeno-graft survival in a pig-to-primate kidney transplant model. Xenotransplantation 2015; 22:221-230. [PubMed: 25847130] $\square$ This study demonstrated that primates with high titers of natural antipig antibody rejected a hCD55/GalTKO kidney xenograft within the first week, as opposed to low-titer animals treated with anti-CD154 antibody which exhibited prolonged (over 4 months) kidney xenograft survival.

4. Iwase H, Liu H, Wijkstrom M, et al. Pig kidney graft survival in a baboon for 136 days: longest lifesupporting organ graft survival to date. Xenotransplantation 2015; 22:302-309. [PubMed: 26130164]

5. Iwase H, Hara H, Ezzelarab M, et al. Immunological and physiological observations in baboons with life-supporting genetically engineered pig kidney grafts. Xenotransplantation 2017; 24. doi: 10.1111/xen.12293. Epub 2017 Mar 17.

6. Tanabe T, Watanabe H, Shah JA, et al. Role of intrinsic (graft) versus extrinsic (host) factors in the growth of transplanted organs following allogeneic and xenogeneic transplantation. Am J Transplant 2017; 17:1778-1790. [PubMed: 28117931] @ Although the authors used a kidney xenotransplant model rather than an XLTx model, by utilizing vascularized donor thymic grafts cotransplanted with kidney from the same donor, the authors achieved greater than 6-month survival of a GalTKO pig thymokidney transplanted into a baboon associated with donor-specific unresponsiveness in vitro.

7. Mohiuddin MM, Singh AK, Corcoran PC, et al. Chimeric 2C10R4 anti-CD40 antibody therapy is critical for long-term survival of GTKO.hCD46.hTBM pigto-primate cardiac xenograft. Nat Commun 2016; 7:11138. [PubMed: 27045379] This study demonstrated long-term heterotopic cardiac xenograft survival for over 2 years using hTBM/hCD46/GalTKO swine combined with their treatment regimen which included anti-CD40.

8. Burdorf L, Laird C, Parsell D, et al. Multitransgenic donor pigs combined with targeted drug treatments extend life-supporting organ function in a xenogeneic lung transplantation model. Transplantation 2016; 100(7S):S446. The abstract was presented at the 26th International Congress of the Transplantation Society; it summarized the results of XLTx using multitransgenic donor pig lungs.

9. Miyata Y, Ohdan H, Yoshioka S, et al. Relationship of xenogeneic micro-chimerism to graft outcome in hamster-to-rat lung xenotransplantation. J Heart Lung Transplant 1998; 17:233-240. [PubMed: 9563599] 
10. Komatsu K, Youm W, Konishi H, et al. Prolonged survival of hamster-to-rat pulmonary xenografts by tacrolimus (FK506) and cyclophosphamide. J Heart Lung Transplant 1996; 15:722-727. [PubMed: 8820789]

11. Nagayasu T, Kawahara K, Takahashi T, et al. Prolongation of lung xenograft survival in rats with a short course of deoxyspergualin and cyclosporin A. Transpl Int 1996; 9:184-193. [PubMed: 8723185]

12. Cooper DK, Satyananda V, Ekser B, et al. Progress in pig-to-nonhuman primate transplantation models (1998-2013): a comprehensive review of the literature. Xenotransplantation 2014; 21:397419. [PubMed: 25176336]

13. Cantu E, Balsara KR, Li B, et al. Prolonged function of macrophage, von Willebrand factordeficient porcine pulmonary xenografts. Am J Transplant 2007; 7:66-75. [PubMed: 17109734]

14. Kim HK, Kim JE, Wi HC, et al. Aurintricarboxylic acid inhibits endothelial activation, complement activation, and von Willebrand factor secretion in vitro and attenuates hyperacute rejection in an ex vivo model of pig-to-human pulmonary xenotransplantation. Xenotransplantation 2008; 15:246-256. [PubMed: 18957047]

15. Holzknecht ZE, Coombes S, Blocher BA, et al. Immune complex formation after xenotransplantation: evidence of type III as well as type II immune reactions provide clues to pathophysiology. Am J Pathol 2001; 158: 627-637. [PubMed: 11159199]

16. Schmelzle M, Schulte Esch J2nd, Robson SC. Coagulation, platelet activation and thrombosis in xenotransplantation. Curr Opin Organ Transpl 2010; 15:212-218.

17. Burdorf L, Riner A, Rybak E, et al. Platelet sequestration and activation during GalTKO.hCD46 pig lung perfusion by human blood is primarily mediated by GPIb, GPIIb/IIIa, and von Willebrand factor. Xenotransplantation 2016; 23:222-236. [PubMed: 27188532]

18. Burdorf L, Azimzadeh AM, Pierson RN3rd. Xenogeneic lung transplantation models. Methods Mol Biol 2012; 885:169-189. [PubMed: 22565996]

19. Nguyen BN, Azimzadeh AM, Schroeder C, et al. Absence of Gal epitope prolongs survival of swine lungs in an ex vivo model of hyperacute rejection. Xenotransplantation 2011; 18:94-107. [PubMed: 21496117]

20. Westall GP, Levvey BJ, Salvaris E, et al. Sustained function of genetically modified porcine lungs in an ex vivo model of pulmonary xenotransplantation. J Heart Lung Transplant 2013; 32:11231130. [PubMed: 23932853]

21. Sahara H, Nagashima H, Miura K, et al. Attenuation of hyperacute dysfunction and microangiopathy by the treatment of carbon monoxide in GalT-KO pulmonary xenotransplantation. Xenotransplantation 2013; 20:359-1359.

22. Burdorf L, Stoddard T, Zhang T, et al. Expression of human CD46 modulates inflammation associated with GalTKO lung xenograft injury. Am J Transplant 2014; 14:1084-1095. [PubMed: 24698431]

23. Esmon CT. The protein C pathway. Chest 2003; 124:26S-32S. [PubMed: 12970121]

24. Roussel JC, Moran CJ, Salvaris EJ, et al. Pig thrombomodulin binds human thrombin but is a poor cofactor for activation of human protein C and TAFI. Am J Transplant 2008; 8:1101-1112. [PubMed: 18444940]

25. Harris DG, Quinn KJ, French BM, et al. Meta-analysis of the independent and cumulative effects of multiple genetic modifications on pig lung xenograft performance during ex vivo perfusion with human blood. Xenotransplantation 2015; 22:102-111. [PubMed: 25470239] $\mathbf{q}$ This article contained a meta-analysis of an ex-vivo xeno-perfusion model of multitransgenic swine lungs. The authors showed that GalTKO and the expression of hCD46, HO-1, hCD55 or hEPCR are associated with improved survival.

26. Burdorf L, Rybak E, Zhang T, et al. Human EPCR expression in GalTKO. hCD46 lungs extends survival time and lowers PVR in a xenogenic lung perfusion model. J Heart Lung Transpl 2013; 32:S137-S1137.

27. Antonioli L, Pacher P, Vizi ES, et al. CD39 and CD73 in immunity and inflammation. Trends Mol Med 2013; 19:355-367. [PubMed: 23601906] 
28. Dwyer KM, Robson SC, Nandurkar HH, et al. Thromboregulatory manifestations in human CD39 transgenic mice and the implications for thrombotic disease and transplantation. J Clin Invest 2004; 113:1440-1446. [PubMed: 15146241]

29. Deaglio S, Robson SC. Ectonucleotidases as regulators of purinergic signaling in thrombosis, inflammation, and immunity. Adv Pharmacol 2011; 61:301-332. [PubMed: 21586363]

30. Motterlini R, Foresti R. Heme oxygenase-1 as a target for drug discovery. Antioxid Redox Signal 2014; 20:1810-1826. [PubMed: 24180608]

31. Lee N, Llano M, Carretero M, et al. HLA-E is a major ligand for the natural killer inhibitory receptor CD94/NKG2A. Proc Natl Acad Sci U S A 1998; 95:5199-5204. [PubMed: 9560253]

32. Laird CT, Burdorf L, French BM, et al. Transgenic expression of human leukocyte antigen-E attenuates GalKO.hCD46 porcine lung xenograft injury. Xenotransplantation 2017; 24. doi: 10.1111/xen.12294. Epub 2017 Mar 3.

33. Nguyen BN, Azimzadeh AM, Zhang T, et al. Life-supporting function of genetically modified swine lungs in baboons. J Thorac Cardiovasc Surg 2007; 133:1354-1363. [PubMed: 17467457]

34. Hassanein W, Braileanu G, Burdorf L, et al. The role of anti-Neu5Gc IgM in xenograft antibody mediated rejection. Am J Transplant 2017; 17(suppl 3): 615.

35. Burdorf L, Rybak E, Zhang T, et al. Combined thromboxane synthase inhibition and H2-receptor blockade prevents PVR elevation during GalTKO. hCD46.hCD55 pig lung perfusion with human blood. J Heart Lung Transpl 2014; 33:S257-S258.

36. Cooper DK, Ekser B, Burlak C, et al. Clinical lung xenotransplantation: what donor genetic modifications may be necessary? Xenotransplantation 2012; 19:144-158. [PubMed: 22702466]

37. Lee W, Miyagawa Y, Long C, et al. Expression of NeuGc on pig corneas and its potential significance in pig corneal xenotransplantation. Cornea 2016; 35:105-113. [PubMed: 26418433]

38. Ekser B, Tector AJ, Cooper DK. Progress toward clinical xenotransplantation. Int J Surg 2015; 23:197-198. [PubMed: 26318503]

39. Burdorf L, Pierson RN III. Preclinical life-supporting lung xenotransplantation: where do we stand? Xenotransplantation 2013; 20:325-326.

40. Bush EL, Barbas AS, Holzknecht ZE, et al. Coagulopathy in alpha-galactosyl transferase knockout pulmonary xenotransplants. Xenotransplantation 2011; 18:6-13. [PubMed: 21342283]

41. Tasaki M, Wamala I, Tena A, et al. High incidence of xenogenic bone marrow engraftment in pigto-baboon intra-bone bone marrow transplantation. Am J Transplant 2015; 15:974-983. [PubMed: 25676635]

42. Sahara H, Shimizu A, Setoyama K, et al. Beneficial effects of perioperative low-dose inhaled carbon monoxide on pulmonary allograft survival in MHC-inbred CLAWN miniature swine. Transplantation 2010; 90:1336-1343. [PubMed: 21076382]

43. Sahara H, Shimizu A, Setoyama K, et al. Carbon monoxide reduces pulmonary ischemiareperfusion injury in miniature swine. J Thorac Cardiovasc Surg 2010; 139:1594-1601. [PubMed: 19909986]

44. Yusen RD, Edwards LB, Kucheryavaya AY, et al. The Registry of the International Society for Heart and Lung Transplantation: thirty-second official adult lung and heart-lung transplantation report-focus theme - early graft failure. J Heart Lung Transplant 2015; 34:1264-1277. [PubMed: 26454740] 


\section{KEY POINTS}

- The striking differences in survival times that are observed between XLTx in small animal models vs. lung grafts in pig to NHP models, as well as the marked improvement in the survival of various solid organ xenografts in pig to NHP models, are all suggestive of the presence of unique immunologic and nonimmunologic barriers that exist in pig to NHP XLTx.

- In order to prevent rapid lung xenograft dysfunction, ex-vivo lung perfusion models as well as in-vivo pig to NHP lung transplant models have been utilized to study the effects of genetically modified pigs along with novel pharmaceutical therapies.

- $\quad$ Because the longest survival with histologically proven viable lung grafts in NHP has been limited to only 5 days, further innovative strategies including the genetic manipulation of donors, as well as the inflammation/coagulation cascade, will be required to make XLTx a clinical possibility. 


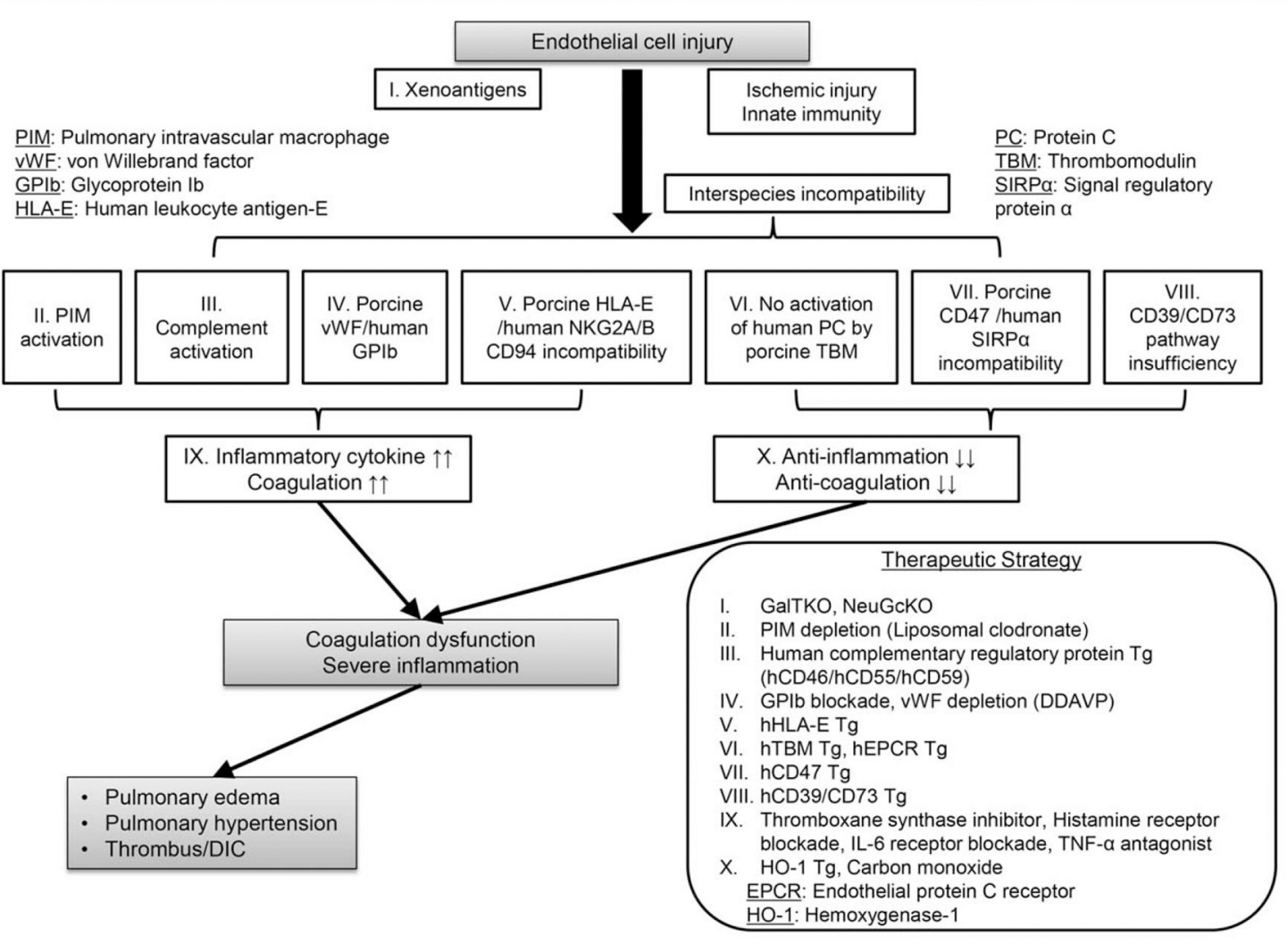

\section{FIGURE 1.}

Strategy to overcome acute pulmonary xenograft dysfunction. The large surface area of pulmonary vascular and alveolar endothelium is thought to be a particularly susceptible target to injury following xeno lung transplantation. Endothelial cell injury after xeno lung transplantation results from severe inflammatory or coagulation dysregulation, which are amplified by the incompatibility between pig and human inflammation/coagulation cascade regulatory proteins, as well as high levels of procoagulant $\mathrm{vWF}$ or proinflammatory pulmonary intravascular macrophages. This ultimately leads to rapid xenogenic lung dysfunction. Several approaches including the use of genetically modified pig lungs, along with pharmaceutical therapies, have been shown to prevent rapid lung xenograft dysfunction. 


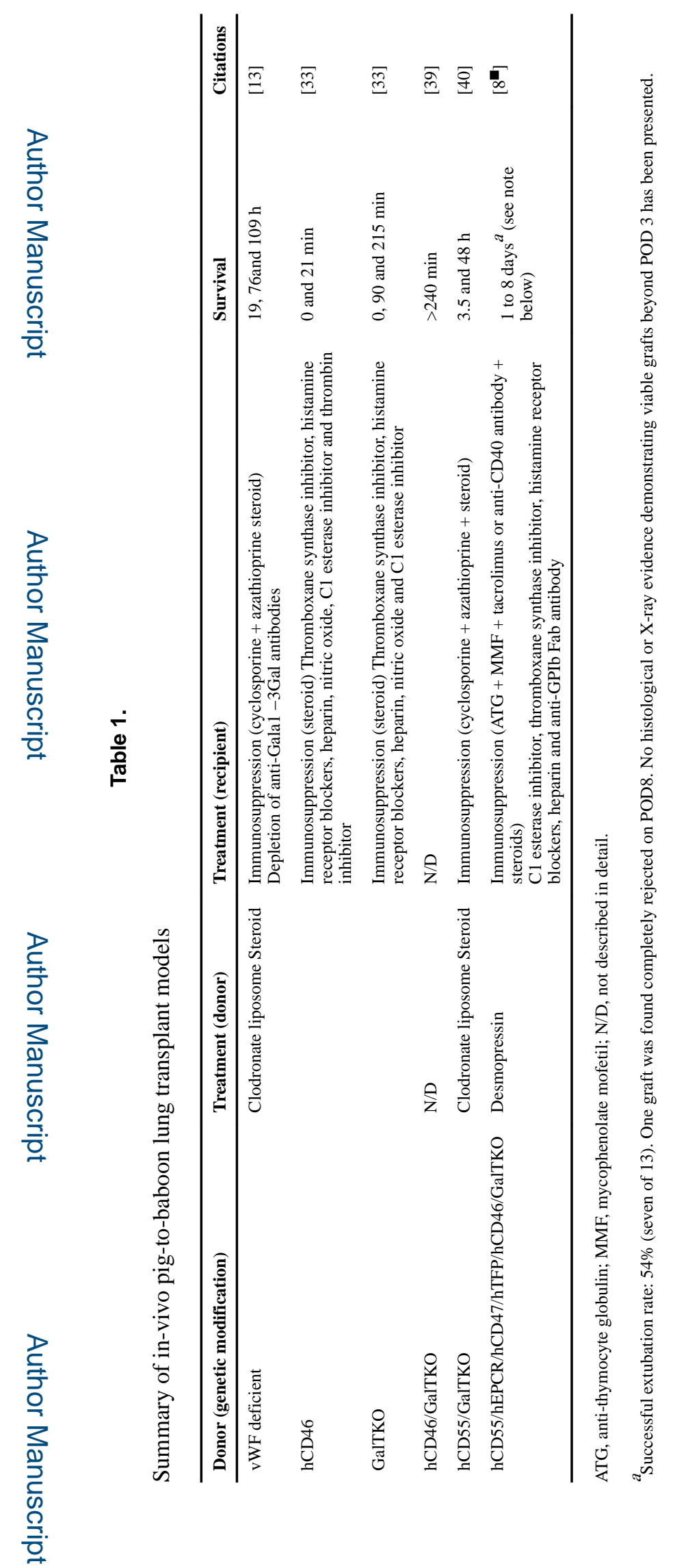

Curr Opin Organ Transplant. Author manuscript; available in PMC 2019 February 27. 